\title{
NEURAL NETWORKS WITH TECHNICAL INDICATORS IDENTIFY BEST TIMING TO INVEST IN THE SELECTED STOCKS
}

\author{
Dr. Asif Ullah Khan ${ }^{1}$ and Dr. Bhupesh Gour ${ }^{2}$ \\ ${ }^{1}$ Professor Dept. of Computer Sc. \& Engineering, TIT , Bhopal \\ asifullahkhan@rediffmail.com \\ ${ }^{2}$ Professor Dept. of Computer Sc. \& Engineering, TIT , Bhopal \\ bhupesh_gourarediffmaol.com
}

\begin{abstract}
Selections of stocks that are suitable for investment are always a complex task. The main aim of every investor is to identify a stock that has potential to go up so that the investor can maximize possible returns on investment. After identification of stock the second important point of decision making is the time to make entry in that particular stock so that investor can get returns on investment in short period of time. There are many conventional techniques being used and these include technical and fundamental analysis. The main issue with any approach is the proper weighting of criteria to obtain a list of stocks that are suitable for investments. This paper proposes an improved method for stock picking and finding entry point of investment that stock using a hybrid method consist of self-organizing maps and selected technical indicators. The stocks selected using our method has given $19.1 \%$ better returns in a period of one month in comparison to SENSEX index.
\end{abstract}

\section{KEYWORDS}

Neural Network, Stocks Classification, Technical Analysis, Fundamental Analysis, SelfOrganizing Map (SOM).

\section{INTRODUCTION}

Selection of stocks that are suitable for investment is a challenging task. Technical Analysis [1] provides a framework for studying investor behaviour, and generally focuses on price and volume data. Technical Analysis using this approach has short-term investment horizons, and access to price and exchange data. Fundamental analysis involves analysis of a company's performance and profitability to determine its share price. By studying the overall economic conditions, the company's competition, and other factors, it is possible to determine expected returns and the intrinsic value of shares. This type of analysis assumes that a share's current (and future) price depends on its intrinsic value and anticipated return on investment. As new information is released pertaining to the company's status, the expected return on the company's shares will change, which affects the stock price. So the advantages of fundamental analysis are its ability to predict changes before they show up on the charts. Growth prospects are related to the current economic environment. Stocks have been selected by us on the basis of fundamental Natarajan Meghanathan et al. (Eds) : ACSIT, FCST, ITCA, CoNeCo - 2015 pp. 25-33, 2014. ( C CS \& IT-CSCP 2015

DOI : $10.5121 /$ csit.2015.51204 
analysis criteria. These criteria are evaluated for each stock and compared in order to obtain a list of stocks that are suitable for investment. Stocks are selected by applying one common criteria on the stocks listed on Bombay Stock Exchange, Mumbai (BSE). The purpose of this paper is to develop a method of classification of selected stocks in to fixed number of classes by Self Organizing map. Each of the class is having its own properties; stocks having properties closer to a particular class get assigned to it. After getting best class stocks we then select stock for investment using technical analysis.

\section{StOCKS Classification}

Stocks are often classified based on the type of company it is, the company's value, or in some cases the level of return that is expected from the company. Some companies grow faster than others, while some have reached what they perceive as their peak and don't think they can handle more growth. In some cases, management just might be content with the level of business that they've achieved, thus stalling to make moves to gain further business. Before investing in a particular company, it is very important to get to know the company on a personal level and find out what the company's goals and objectives are for the short and long term. In order to prosper in the world of stock investing, a person must have a clear understanding of what they are doing, or they shouldn't be doing it at all. Stocks can be a very risky investment, depending on the level of knowledge held by the person(s) making the investment decisions. Below is a list of classifications which are generally known to us- Growth Stocks, Value Stocks, Large Cap Stocks, Mid Cap Stocks, and Small Cap Stocks. Stocks are usually classified according to their characteristics. Some are classified according to their growth potential in the long run and the others as per their current valuations. Similarly, stocks can also be classified according to their market capitalization. The classifications are not rigid and no rules are laid down anywhere for their classification. We classified stocks by taking in account the Shareholding Pattern, P/E Ratio, Dividend Yield, Price/Book Value Ratio, Return on Net worth (RONW), Annual growth in Sales, Annual growth in Reported Profit After Tax, Return on Capital Employed (ROCE) and Adjusted Profit After Tax Margin (APATM) with Self-Organizing Map.

\section{STOCK MARKET INDEX}

A stock market index is a method of measuring a stock market as a whole. Stock market indexes may be classed in many ways. A broad-base index represents the performance of a whole stock market - and by proxy, reflects investor sentiment on the state of the economy. The most regularly quoted market indexes are broad-base indexes comprised of the stocks of large companies listed on a nation's largest stock exchanges, such as the American Dow Jones Industrial Average and S\&P 500 Index, the British FTSE 100, the French CAC 40, the German DAX, the Japanese Nikkei 225, the Indian Sensex and the Hong Kong Hang Seng Index. Movements of the index should represent the returns obtained by "typical" portfolios in the country. Ups and downs in the index reflect the changing expectations of the stock market about future dividends of country's corporate sector. When the index goes up, it is because the stock market thinks that the prospective dividends in the future will be better than previously thought. When prospects of dividends in the future become pessimistic, the index drops. 


\subsection{COMPOSITION OF STOCK MARKET INDEX}

The most important type of market index is the broad-market index, consisting of the large, liquid stocks of the country. In most countries, a single major index dominates benchmarking, index funds, index derivatives and research applications. In addition, more specialised indices often find interesting applications. In India, we have seen situations where a dedicated industry fund uses an industry index as a benchmark. In India, where clear categories of ownership groups exist, it becomes interesting to examine the performance of classes of companies sorted by ownership group. We compared BSE-30 SENSEX with the stock selected using SOM and GABPN. We choose BSE-30 SENSEX for comparison because SENSEX is regarded to be the pulse of the Indian stock market. As the oldest index in the country, it provides the time series data over a fairly long period of time (From 1979 onwards). Small wonder, the SENSEX has over the years become one of the most prominent brands in the country. SENSEX is calculated using the "Free-float Market Capitalization" methodology. As per this methodology, the level of index at any point of time reflects the free-float market value of 30 component stocks relative to a base period. The market capitalization of a company is determined by multiplying the price of its stock by the number of shares issued by the company. This market capitalization is further multiplied by the free-float factor to determine the free-float market capitalization. The base period of SENSEX is 1978-79 and the base value is 100 index points. This is often indicated by the notation 1978-79=100. The calculation of SENSEX involves dividing the Free-float market capitalization of 30 companies in the Index by a number called the Index Divisor. The Divisor is the only link to the original base period value of the SENSEX. It keeps the Index comparable over time and is the adjustment point for all Index adjustments arising out of corporate actions, replacement of scrips etc. During market hours, prices of the index scrips, at which latest trades are executed, are used by the trading system to calculate SENSEX every 15 seconds and disseminated in real time.

Table 1: List of companies of SENSEX

\begin{tabular}{|l|l|}
\hline SENSEX & BAJAJ AUTO, BHARTI AIRTEL, \\
& BHEL, CIPLA, COAL INDIA, \\
DRREDDY, GAIL, HDFC, HDFCBANK, & HEROMOTORCO, HINDALCO, HUL, \\
ICICIBANK, INFY, ITC, \\
JINDALSTEEL, LNT, MARUTI, MNM, \\
NTPC, ONGC, RIL, SBI, \\
STERLITEIND, SUNPHARMA, \\
TATAMOTORS, TATAPOWER, \\
TATASTL, TCS, WIPRO \\
\end{tabular}

\section{APPLICATION OF NEURAL NETWORKS IN STOCKS}

\subsection{Overview}

The ability of neural networks to discover nonlinear relationships [3] in input data makes them ideal for modeling nonlinear dynamic systems such as the stock market. Neural networks, with 
their remarkable ability to derive meaning from complicated or imprecise data, can be used to extract patterns and detect trends that are too complex to be noticed by either humans or other computer techniques. A neural network method can enhance an investor's forecasting ability [4]. Neural networks are also gaining popularity in forecasting market variables [5]. A trained neural network can be thought of as an expert in the category of information it has been given to analyze. This expert can then be used to provide projections given new situations of interest and answer "what if" questions. Traditionally forecasting research and practice had been dominated by statistical methods but results were insufficient in prediction accuracy [6]. Monica et al's work [7] supported the potential of NNs for forecasting and prediction. Asif Ullah Khan et al. [8] used the back propagation neural networks with different number of hidden layers to analyze the prediction of the buy/sell. Neural networks using back propagation algorithms having one hidden layer give more accurate results in comparison to two, three, four and five hidden layers.

\subsection{Kohonen self-organizing map}

Self-organizing maps (SOM) belong to a general class of neural network methods, which are nonlinear regression techniques that can be applied to find relationships between inputs and outputs or organize data so as to disclose so far unknown patterns or structures. It is an excellent tool in exploratory phase of data mining [9]. It is widely used in application to the analysis of financial information [10]. The results of the study indicate that self-organizing maps can be feasible tools for classification of large amounts of financial data [11]. The Self-Organizing Map, SOM, has established its position as a widely applied tool in data-analysis and visualization of high-dimensional data. Within other statistical methods the SOM has no close counterpart, and thus it provides a complementary view to the data. The SOM is, however, the most widely used method in this category, because it provides some notable advantages over the alternatives. These include, ease of use, especially for inexperienced users, and very intuitive display of the data projected on to a regular two-dimensional slab, as on a sheet of a paper. The main potential of the SOM is in exploratory data analysis, which differs from standard statistical data analysis in that there are no presumed set of hypotheses that are validated in the analysis. Instead, the hypotheses are generated from the data in the data-driven exploratory phase and validated in the confirmatory phase. There are some problems where the exploratory phase may be sufficient alone, such as visualization of data without more quantitative statistical inference upon it. In practical data analysis problems the most common task is to search for dependencies between variables. In such a problem, SOM can be used for getting insight to the data and for the initial search of potential dependencies. In general the findings need to be validated with more classical methods, in order to assess the confidence of the conclusions and to reject those that are not

statistically significant. In this contribution we discuss the use of the SOM in searching for dependencies in the data. First we normalize the selected parameters and then we initialize the SOM network. We then train SOM to give the maximum likelihood estimate, so that we can associate a particular stock with a particular node in the classification layer. The self-organizing networks assume a topological structure among the cluster units [2]. There are $\mathrm{m}$ cluster units, arranged in a one or two dimensional array: the input signals are n-dimensional. Fig. 1 shows architecture of self-organizing network (SOM), which consists of input layer, and Kohonen or clustering layer. 


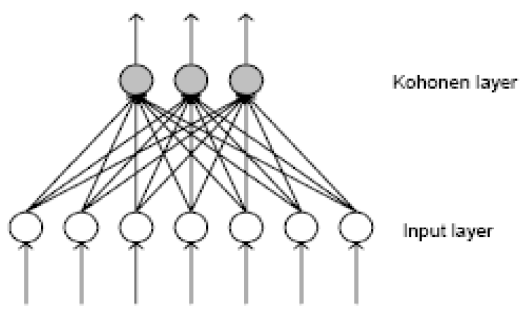

Figure.1: Architecture of Kohonen self-organizing map

The shadowed units in the Fig. 1 are processing units. SOM network may cluster the data into N number of classes. When a self-organizing network is used, an input vector is presented at each step. These vectors constitute the "environment" of the network. Each new input produces an adaptation of the parameters. If such modifications are correctly controlled, the network can build a kind of internal representation of the environment.

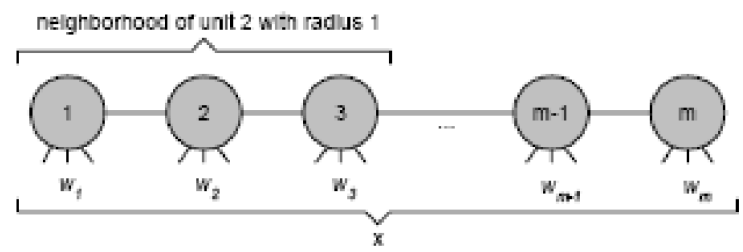

Fig. 2: A one-dimensional lattice of computing units.

The n-dimensional weight vectors $w_{1}, w_{2}, \ldots, w_{m}$ are used for the computation. The objective of the clustering for each unit is to learn the specialized pattern present on different regions of input space as shown in Fig. 2. When an input from such a region is fed into the network, the corresponding unit should compute the maximum excitation. SOM may distinctly reduce misclassification errors [12]. Kohonen's learning algorithm is used to guarantee that this effect is achieved. A Kohonen unit computes the Euclidian distance between an input $x$ and its weight vector $w$. The complete description of Kohonen learning algorithm can be found in [2] and [3].

\section{TECHNICAL ANALYSIS}

Technical analysis is a method of evaluating securities by analyzing the statistics generated by market activity, such as past prices and volume. Technical analysts do not attempt to measure a security's intrinsic value, but instead use charts and other tools to identify patterns that can suggest future activity. Just as there are many investment styles on the fundamental side, there are also many different types of technical traders. Some rely on chart patterns; others use technical indicators and oscillators, and most use some combination of the two. In any case, technical analysts' exclusive use of historical price and volume data is what separates them from their fundamental counterparts. Unlike fundamental analysts, technical analysts don't care whether a stock is undervalued - the only thing that matters is a security's past trading data and what information this data can provide about where the security might move in the future. The field of technical analysis is based on three assumptions:

1. The market discounts everything.

2. Price moves in trends

3. History tends to repeat itself. 
Despite all the fancy and exotic tools it employs, technical analysis really just studies supply and demand in a market in an attempt to determine what direction, or trend, will continue in the future. In other words, technical analysis attempts to understand the emotions in the market by studying the market itself, as opposed to its components. Moving Average, MACD, ROC and RSI are mostly used technical indicators.

\subsection{RSI}

The name "Relative Strength Index" is slightly misleading as the RSI does not compare the relative strength of two securities, but rather the internal strength of a single security. A more appropriate name might be "Internal Strength Index". The RSI usually tops above 70 and bottoms below 30. It usually forms these tops and bottoms before the underlying price chart.9-day RSI is used for calculation.

\subsection{Williams \% R}

Williams $\% \mathrm{R}$ is a momentum indicator that is the inverse of the Fast Stochastic Oscillator. Also referred to as $\% \mathrm{R}$, Williams $\% \mathrm{R}$ reflects the level of the close relative to the highest high for the look-back period. In contrast, the Stochastic Oscillator reflects the level of the close relative to the lowest low. $\% \mathrm{R}$ corrects for the inversion by multiplying the raw value by -100 . As a result, the Fast Stochastic Oscillator and Williams \% R produce the exact same lines, only the scaling is different. Williams $\% \mathrm{R}$ oscillates from 0 to -100 . Readings from 0 to -20 are considered overbought. Readings from -80 to -100 are considered oversold. Unsurprisingly, signals derived from the Stochastic Oscillator are also applicable to Williams \%R.

\subsection{Ultimate Oscillator}

Ultimate Oscillator is a momentum oscillator designed to capture momentum across three different timeframes. The multiple timeframe objective seeks to avoid the pitfalls of other oscillators. Many momentum oscillators surge at the beginning of a strong advance and then form bearish divergence as the advance continues. This is because they are stuck with one time frame. The Ultimate Oscillator attempts to correct this fault by incorporating longer timeframes into the basic formula. Williams identified a buy signal based on a bullish divergence and a sell signal based on a bearish divergence.

\subsection{MACD}

It is based on 3 exponential moving averages, or EMA. These averages can be of any period, though the most common combination, and the one we have focused on, is the 12-26-9 days MACD. If the MACD is above the 9-days EMA buy signal is generated and If MACD is below the 9-days EMA sell signal is generated

\subsection{Stochastic Oscillator}

The Stochastic Oscillator is a momentum indicator that shows the location of the close relative to the high-low range over a set number of periods.The Stochastic Oscillator "doesn't follow price, it doesn't follow volume or anything like that. It follows the speed or the momentum of price. As a rule, the momentum changes direction before price." As such, bullish and bearish divergences 
in the Stochastic Oscillator can be used to foreshadow reversals. This was the first, and most important, signal that Lane identified. Lane also used this oscillator to identify bull and bear setups to anticipate a future reversal. Because the Stochastic Oscillator is range bound, is also useful for identifying overbought and oversold levels.

\subsection{On Balance Volume (OBV)}

On Balance Volume (OBV) measures buying and selling pressure as a cumulative indicator that adds volume on up days and subtracts volume on down days. OBV was developed by Joe Granville. It was one of the first indicators to measure positive and negative volume flow. Chartists can look for divergences between OBV and price to predict price movements or use OBV to confirm price trends.

\section{EXPERIMENTAL RESULTS}

The system has been developed and tested on Windows XP operating system .We have used Visual Basic and Microsoft Access as front end and back end tool. Simulation data was sourced from Indian Bombay Stock Exchange (BSE).We have selected technical indicators RSI, Williams \%R, Ultimate Oscillator, MACD, Stochastic Oscillator, On Balance Volume (OBV). With these inputs SOM divides them into different classes. As the SOM are more relevant to the problem where stocks of different companies are to be compared on some common parameters and arranges in the form of different classes. Out of these classes we compared stocks belonging to the best class with the above specified technical indicators. Input attributes should be carefully selected to keep the dimensionality of input vectors relatively small [16]. As we know close rates are primary quantitative factors for individual equities and from quantitative factors the key qualitative factor of the market sentiment can be derived. So we used close rate of stocks as our input in the technical indicators. Stocks classified using SOM and then selected by technical indicators is compared with BSE-30 index for the period 20/07/2009 to 20/08/2009. We have found that our selected stock gives $19.1 \%$ more returns in comparison to BSE-30 Index as shown in fig. 3.

Table 2: Buy and Sell rates of Selected Stocks and Sensex

\begin{tabular}{|c|c|c|c|c|c|c|c|}
\hline \multirow[b]{2}{*}{ SN } & \multirow[b]{2}{*}{ sTock } & \multicolumn{3}{|c|}{ BUY } & \multicolumn{3}{|c|}{ SELL } \\
\hline & & DATE & RATE & SEHSEX & DATE & RATE & SENSEX \\
\hline 1 & GMR & $27108+12$ & 18.3 & 17678.8 & $27 / 09 / 12$ & 24.1 & 18579.5 \\
\hline 2 & HDIL & $03109+12$ & 66.95 & 17384.4 & $03 / 10 / 12$ & 100.9 & 18869.7 \\
\hline 3 & RFOWER & $31+08 / 12$ & 76.2 & 17380.8 & $01 / 10 / 12$ & 98.5 & 18823.9 \\
\hline 4 & JP ASSO & $29 r 09+12$ & 64 & 17490.8 & $01 / 10 / 12$ & 86.0 & 18823.9 \\
\hline
\end{tabular}


Table 3: Comparison of Selected Stocks and Sensex

\begin{tabular}{|c|c|c|c|c|}
\hline \multirow[b]{2}{*}{ SN } & \multirow[b]{2}{*}{ STOCK } & \multicolumn{2}{|c|}{ GAIN (\%) } & \multirow{2}{*}{\begin{tabular}{|c} 
DIFF. IN \\
GAIN \\
\end{tabular}} \\
\hline & & STOCK & SENSEX & \\
\hline 1 & GMR & 24.1 & 5.1 & 19.0 \\
\hline 2 & HDIL & 33.6 & 8.5 & 25.1 \\
\hline 3 & RPOWER & 22.6 & 8.3 & 14.3 \\
\hline 4 & IP ASSO & 25.5 & 7.6 & 17.9 \\
\hline \multicolumn{2}{|c|}{ AVERAGE } & 26.4 & 7.3 & 19.1 \\
\hline
\end{tabular}

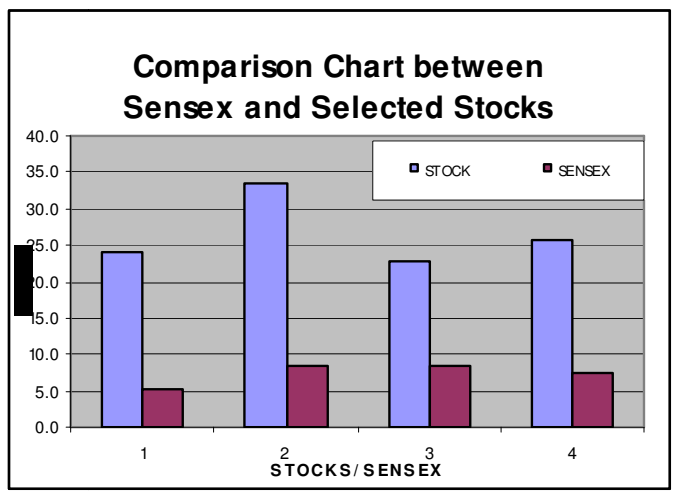

Figure. 3: Comparison chart between stocks selected using SOM and technical indicators with BSE-30 Index.

\section{CONCLUSION}

This paper compares the performances of the stock selected by using hybrid model of SelfOrganizing Maps and technical indicators with BSE-30 Index. The stocks selected by Hybrid model of SOM and technical indicators help the investor not only in selecting stocks but also in identifying the timing of purchasing the particular stock. The result shows that the performance of stocks belonging to the best class among the classes generated by self-organizing maps and then stock selected using Technical Indicators gives better returns on investment. Stock selected using SOM and Technical Indicators gives $28.41 \%$ more returns in comparison to BSE-30 Index.

\section{REFERENCES}

[1] Mizuno, H., Kosaka, M., Yajima, H. and Komoda N., "Application of Neural Network to Technical Analysis of Stock Market Prediction", Studies in Informatic and Control, 1998, Vol.7, No.3, pp.111120.

[2] Haykin, Simon, "Neural Networks: A Comprehensive Foundation", Macmillian College Publishing Company, New York, 1994.

[3] Phillip D. Wasserman, Van Nostrand, "Neural Computing: Theory and Practice", Van Nostrand Reinhold, New York, 1989.

[4] Youngohc yoon and George swales, "Predicting stock price performance: a neural network approach", IEEE publishing, 1991.

[5] Shaikh A. Hamid, "Primer on using neural networks for forecasting market variables", in proceedings of the conference at school of business, Southern New Hampshire university, 2004. 
[6] Ramon Lawrence, "Using Neural Networks to Forecast Stock Market Prices", Course Project, University of Manitoba Dec. 12, 1997.

[7] Monica Adya and Fred Collopy, "How Effective are Neural Networks at Forecasting and Prediction? A Review and Evaluation", Journal of Forecasting, 1998.

[8] Asif Ullah Khan et al., "Stock Rate Prediction Using Back Propagation Algorithm: Analyzing the prediction accuracy with different number of hidden layers", Glow gift, Bhopal, 2005.

[9] Juha Vesanto and Esa Alhoniemi, "Clustering of the Self-Organizing Map", IEEE Transactions on Neural Networks, Vol. 11, No. 3, May 2000.

[10] Serrano, C., "Self Organizing Neural Networks for Financial Diagnosis", Decision Support Systems Elsevier Science, 1996, Vol 17, July, pp. 227-238.

[11] Tomas Eklund, "Assesing the feasibility of self organizing maps for data mining financial information", ECIS, June 6-8, 2002, Gdansk, Poland.

[12] Egidijus Merkevicius, Gintautas Garsva, "Forecasting of credit classes with the self organizing maps", Informacines Technologies (ISSN 1392 - 124X) Ir Valdymas, 2004, Nr.4(33).

[13] D. E. Goldberg, "Genetic Algorithms in Search, Optimization and Machine Learning." New York: Addison-Wesley, 1989.

[14] K. Bergerson and D. Wunsch, "A commodity trading model based on a neural network- expert system hybrid", IJCNN-91- Seattle International Joint Conference, Volume I, Issue 8-14 Jul 1991, Page(s): $289-293$.

[15] Asif Ullah Khan et al., " Comparisons of Stock Rates Prediction Accuracy using Different Technical Indicators with Backpropagation Neural Network and Genetic Algorithm Based Backpropagation Neural Network", pp. 575-580, 978-0-7695-3267-7/08 \$25.00 (C) 2008 IEEE DOI 10.1109/ICETET.2008.59.

[16] H. White, "Economic prediction using neural networks: The case of IBM daily stock returns", in Neural Networks in Finance and Investing, chapter18, pages 315-328, 1993. 